\title{
The DMO Sum Rule Revisited
}

\author{
Eugene Golowich \\ Department of Physics and Astronomy, University of Massachusetts \\ Amherst MA 01003 USA \\ Joachim Kambor \\ Institut für Theoretische Physik, Universität Zürich \\ CH-8057 Zürich, Switzerland
}

\begin{abstract}
We reconsider the DMO sum rule in light of our recent two-loop calculations of isospin and hypercharge vector and axialvector current propagators in chiral perturbation theory. A modified derivation valid to second order in the light quark masses is presented, and a phenomenological analysis yields determination of a combination of finite counterterms occurring in the $p^{4}$ and $p^{6}$ chiral lagrangians. Suggestions are given for further study.
\end{abstract}

Working in the chiral limit of massless quarks, Weinberg was the first to derive spectral function sum rules involving the vector and axialvector currents. [1] There soon followed the announcement of two additional chiral sum rules, one for the pion electromagnetic mass difference [2] and one (the DMO sum rule [3]) involving an inverse moment of chiral spectral functions, both also derived in the chiral limit. A subsequent study of chiral sum rules in QCD determined that only the first Weinberg sum rule and the DMO sum rule survive the inclusion of quark mass effects. [4, 5] About a decade later, the DMO sum rule was analyzed by applying the Gasser-Leutwyler analysis of chiral perturbation theory (ChPT) at oneloop order. [6 8] In this paper, we report on a re-analysis of the DMO sum rule based on our recent calculations of vector and axialvector current propagators at two-loop order in ChPT. [9, 10]

\section{Derivation of DMO Sum Rule in Two-Loop ChPT}

Given the normalization of vector and axialvector octet flavour currents,

$$
J_{\mathrm{V} i}^{\mu}=\bar{q} \frac{\lambda_{i}}{2} \gamma^{\mu} q \quad \text { and } \quad J_{\mathrm{A} i}^{\mu}=\bar{q} \frac{\lambda_{i}}{2} \gamma^{\mu} \gamma_{5} q \quad(i=1, \ldots, 8)
$$


one defines the isospin vector and axialvector current propagators respectively as

$$
\Delta_{\mathrm{k} 3}^{\mu \nu}\left(q^{2}\right) \equiv i \int d^{4} x e^{i q \cdot x}\left\langle 0\left|T\left(J_{\mathrm{k} 3}^{\mu}(x) J_{\mathrm{k} 3}^{\nu}(0)\right)\right| 0\right\rangle \quad(\mathrm{k}=\mathrm{V}, \mathrm{A}) .
$$

These have the tensorial decompositions

$$
\Delta_{\mathrm{k} 3}^{\mu \nu}\left(q^{2}\right)=\left(q^{\mu} q^{\nu}-q^{2} g^{\mu \nu}\right) \Pi_{\mathrm{k} 3}^{(1)}\left(q^{2}\right)+q^{\mu} q^{\nu} \Pi_{\mathrm{k} 3}^{(0)}\left(q^{2}\right) \quad(\mathrm{k}=\mathrm{V}, \mathrm{A}),
$$

and spectral functions obtained via imaginary parts,

$$
\frac{1}{\pi} \mathcal{I} m \Delta_{\mathrm{k} 3}^{\mu \nu}\left(q^{2}\right)=\left(q^{\mu} q^{\nu}-q^{2} g^{\mu \nu}\right) \rho_{\mathrm{k} 3}^{(1)}\left(q^{2}\right)+q^{\mu} q^{\nu} \rho_{\mathrm{k} 3}^{(0)}\left(q^{2}\right) \quad(\mathrm{k}=\mathrm{V}, \mathrm{A}) .
$$

In the following we shall assume isospin symmetry, for which the spin-zero vector current contributions vanish $\left(\Pi_{\mathrm{V} 3}^{(0)}=0\right.$ and $\left.\rho_{\mathrm{V} 3}^{(0)}=0\right)$.

The asymptotic behavior $(s \rightarrow \infty)$ of the vector and axialvector spectral functions predicted by QCD is given as [4], [5], [11]

$$
\left(\rho_{\mathrm{V} 3}^{(1)}-\rho_{\mathrm{A} 3}^{(1)}-\rho_{\mathrm{A} 3}^{(0)}\right)(s) \sim o\left(s^{-1}\right) .
$$

The information in Eq. (5) can be used, together with analyticity and the corresponding asymptotic behavior of the polarization functions, to derive dispersion relations for the vector and axialvector polarization functions,

$$
\left(\Pi_{\mathrm{V} 3}^{(1)}-\Pi_{\mathrm{A} 3}^{(1)}-\Pi_{\mathrm{A} 3}^{(0)}\right)\left(q^{2}\right)=\int_{0}^{\infty} d s \frac{\left(\rho_{\mathrm{V} 3}^{(1)}-\rho_{\mathrm{A} 3}^{(1)}-\rho_{\mathrm{A} 3}^{(0)}\right)(s)}{s-q^{2}-i \epsilon} .
$$

Upon evaluation at $q^{2}=0$, Eq. (3) yields the inverse-moment DMO (or ' $L_{10}$ ') sum rule [3],

$$
\left(\Pi_{\mathrm{V} 3}^{(1)}-\Pi_{\mathrm{A} 3}^{(1)}-\Pi_{\mathrm{A} 3}^{(0)}\right)(0)=\int_{0}^{\infty} d s \frac{\left(\rho_{\mathrm{V} 3}^{(1)}-\rho_{\mathrm{A} 3}^{(1)}-\rho_{\mathrm{A} 3}^{(0)}\right)(s)}{s} .
$$

For the purpose of phenomenological analysis, one wishes the spectral integral to contain only quantities which have already been measured. Therefore, we make use of the ChPT analysis of Ref. [12 to cast the DMO sum rule in the optimal form,

$$
\left(\Pi_{\mathrm{V} 3}^{(1)}-\hat{\Pi}_{\mathrm{A} 3}^{(1)}+\frac{d \hat{\Pi}_{\mathrm{A} 3}^{(0)}}{d q^{2}}\right)(0)+4 H_{1}^{(0)}(\mu)-2 L_{10}^{(0)}(\mu)=\int_{0}^{\infty} d s \frac{\left(\rho_{\mathrm{V} 3}^{(1)}-\rho_{\mathrm{A} 3}^{(1)}\right)(s)}{s},
$$

where $H_{1}^{(0)}(\mu)$ and $L_{10}^{(0)}(\mu)$ are counterterms from the $p^{4}$ chiral lagrangian and $\hat{\Pi}_{\mathrm{A} 3}^{(1,0)}$ are finite polarization functions entering at two-loop order [10,12].

\section{ChPT Contributions to DMO Sum Rule}

From Refs. [9,10], we obtain the following expressions for terms appearing on the lefthand-side (LHS) of Eq. (8),

$$
\text { LHS }=G^{(1)}+G^{(2)} \equiv G^{(1)}+G_{\pi}^{(2)}+G_{K}^{(2)}+G_{\mathrm{NUM}}^{(2)}+G_{\mathrm{CT}}^{(2)},
$$


with

$$
\begin{aligned}
& G^{(1)} \equiv-4 L_{10}^{(0)}(\mu)-\frac{1}{48 \pi^{2}} \log \frac{M_{\pi}^{2}}{\mu^{2}}-\frac{1}{96 \pi^{2}} \log \frac{M_{K}^{2}}{\mu^{2}}-\frac{1}{32 \pi^{2}}, \\
& G_{\pi}^{(2)} \equiv \frac{M_{\pi}^{2}}{\pi^{4} F_{\pi}^{2}}\left[\log \frac{M_{\pi}^{2}}{\mu^{2}}\left(-\frac{1}{288}+\frac{1}{768} \log \frac{M_{\pi}^{2}}{\mu^{2}}+\frac{1}{768} \log \frac{M_{K}^{2}}{\mu^{2}}+\pi^{2} L_{10}^{(0)}(\mu)+\frac{\pi^{2}}{2} L_{9}^{(0)}(\mu)\right)\right. \\
&\left.\quad+\log \frac{M_{K}^{2}}{\mu^{2}}\left(-\frac{1}{576}-\frac{1}{1536} \log \frac{M_{K}^{2}}{\mu^{2}}\right)\right], \\
& G_{K}^{(2)} \equiv \frac{M_{K}^{2}}{\pi^{4} F_{\pi}^{2}} \log \frac{M_{K}^{2}}{\mu^{2}}\left[-\frac{17}{3072}+\frac{1}{1024} \log \frac{M_{K}^{2}}{\mu^{2}}+\frac{\pi^{2}}{2} L_{10}^{(0)}(\mu)+\frac{\pi^{2}}{4} L_{9}^{(0)}(\mu)\right], \\
& G_{\mathrm{NUM}}^{(2)} \equiv-\frac{49}{13824 \pi^{4}} \frac{M_{\pi}^{2}}{F_{\pi}^{2}}-\frac{5}{36864 \pi^{4}} \frac{M_{K}^{2}}{F_{\pi}^{2}}-\frac{1.927 \cdot 10^{-6} \mathrm{GeV}^{2}}{F_{\pi}^{2}}+\frac{4.132 \cdot 10^{-9} \mathrm{GeV}^{2}}{F_{\pi}^{2}}, \\
& G_{\mathrm{CT}}^{(2)} \equiv \frac{4 M_{\pi}^{2}}{F_{\pi}^{2}}\left(Q_{\mathrm{A}}^{\overline{\mathrm{MS}}}(\mu)+R_{\mathrm{A}}^{\overline{\mathrm{MS}}}(\mu)-Q_{\mathrm{V}}^{\overline{\mathrm{MS}}}(\mu)-R_{\mathrm{V}}^{\overline{\mathrm{MS}}}(\mu)\right)+\frac{8 M_{K}^{2}}{F_{\pi}^{2}}\left(R_{\mathrm{A}}^{\overline{\mathrm{MS}}}(\mu)-R_{\mathrm{V}}^{\overline{\mathrm{MS}}}(\mu)\right),
\end{aligned}
$$

where $G^{(1)}$ and $G^{(2)}$ are respectively the ChPT one-loop and two-loop contributions and the final two (numerical) terms in $G_{\mathrm{NUM}}^{(2)}$ arise from the so-called sunset amplitudes. All the two-loop quantities are new contributions which have not previously appeared in the literature. We shall discuss the counterterm contributions separately in a later section.

\section{Phenomenological Determination of DMO Spectral Integral}

One anticipates an accurate determination of the DMO spectral integral

$$
I_{\mathrm{DMO}} \equiv \int_{0}^{\infty} d s \frac{\left(\rho_{\mathrm{V} 3}^{(1)}-\rho_{\mathrm{A} 3}^{(1)}\right)(s)}{s}
$$

insofar as the inverse factor of the squared-energy suppresses contributions at large $s$. The dominant contributions to $I_{\mathrm{DMO}}$ will arise from the $2 \pi$ and $3 \pi$ resonances $\rho(770)$ and $a_{1}(1260)$, with smaller contributions from $n_{\pi} \geq 4$ multipion states, $\bar{K} K$-multipion states, etc. One can access such information via studies of tau-lepton decay and $e^{+} e^{-} \rightarrow 2 \pi$, etc cross sections. [13]

An evaluation of $I_{\mathrm{DMO}}$ which employs as input the most recent $\tau$ and $e^{+} e^{-}$data available and which neglects contributions from $s>m_{\tau}^{2}$ yields 14

$$
I_{\mathrm{DMO}}=(26.4 \pm 1.5) \cdot 10^{-3},
$$

with an uncertainty of about 5.7\%. In a modified analysis, one defines the laplace transformed quantity

$$
\hat{I}_{\mathrm{DMO}}\left(M^{2}\right) \equiv \int_{0}^{\infty} d s e^{s / M^{2}} \frac{\left(\rho_{\mathrm{V} 3}^{(1)}-\rho_{\mathrm{A} 3}^{(1)}\right)(s)}{s}+\frac{F_{\pi}^{2}}{M^{2}}-\frac{C_{6} \mathcal{O}_{6}}{6 M^{6}}-\frac{C_{8} \mathcal{O}_{8}}{24 M^{8}}
$$

where $I_{\mathrm{DMO}}=\lim _{M \rightarrow \infty} \hat{I}_{\mathrm{DMO}}\left(M^{2}\right)$ and the final two terms involve nonperturbative contributions. A fit to several $M^{2}$-moments then yields [14]

$$
I_{\mathrm{DMO}}=(25.8 \pm 0.3 \pm 0.1) \cdot 10^{-3}
$$

with a claimed uncertainty of about $1.24 \%$. 


\section{Inputs to Counterterm Contributions}

Consider the contributions in Eq. (9) arising from counterterms. There are those from the chiral lagrangians of order $p^{4}\left(L_{9}^{(0)}\right.$ and $\left.L_{10}^{(0)}\right)$ and those from lagrangians of order $p^{6}$ $\left(Q_{\mathrm{A}}^{\overline{\mathrm{MS}}}, R_{\mathrm{A}}^{\overline{\mathrm{MS}}}, Q_{\mathrm{V}}^{\overline{\mathrm{MS}}}\right.$ and $\left.R_{\mathrm{V}}^{\overline{\mathrm{MS}}}\right)$. All these counterterms are evaluated in $\overline{\mathrm{MS}}$ renormalization.円 For notational consistency with the axial propagator counterterms, the vector propagator counterterms, defined earlier in Refs. [9, 15] as $Q$ and $R$, are denoted here respectively as $Q_{\mathrm{V}}^{\overline{\mathrm{MS}}}$ and $R_{\mathrm{V}}^{\overline{\mathrm{MS}}}$.

The apparent dependence in Eq. (91) on the scale $\mu$ is illusory. The explicit scale dependence of the logarithmic terms is compensated by implicit scale dependence in the $p^{4}$ and $p^{6}$ counterterms such that the LHS is independent of scale. In particular, the scale dependence of the $p^{6}$ counterterms is

$$
\begin{aligned}
& \left(Q_{\mathrm{A}}^{\overline{\mathrm{MS}}}(\mu)-Q_{\mathrm{V}}^{\overline{\mathrm{MS}}}(\mu)\right)-\left(\mu \rightarrow \mu_{0}\right)= \\
& \quad \frac{1}{\left(16 \pi^{2}\right)^{2}} \log \frac{\mu_{0}^{2}}{\mu^{2}}\left[\frac{5}{32}-\frac{3}{2}\left(L_{9}^{(0)}\left(\mu_{0}\right)+L_{10}^{(0)}\left(\mu_{0}\right)\right)+\frac{3}{32} \log \frac{\mu_{0}^{2}}{\mu^{2}}\right], \\
& \left(R_{\mathrm{A}}^{\overline{\mathrm{MS}}}(\mu)-R_{\mathrm{V}}^{\overline{\mathrm{MS}}}(\mu)\right)-\left(\mu \rightarrow \mu_{0}\right)= \\
& \quad \frac{1}{\left(16 \pi^{2}\right)^{2}} \log \frac{\mu_{0}^{2}}{\mu^{2}}\left[\frac{17}{96}-\frac{1}{2}\left(L_{9}^{(0)}\left(\mu_{0}\right)+L_{10}^{(0)}\left(\mu_{0}\right)\right)+\frac{1}{32} \log \frac{\mu_{0}^{2}}{\mu^{2}}\right],
\end{aligned}
$$

as can be obtained from renormalization group equations [9].

The collection of $p^{4}$ and $p^{6}$ counterterms originate from the renormalization procedure, and each must ultimately be determined from experiment. The $p^{4}$ counterterms $L_{9}^{(0)}$ and $L_{10}^{(0)}$ are already known [16] from one-loop analyses to accuracies respectively of about $10 \%$ and $13 \%$,

$$
\begin{array}{ll}
L_{9}^{(0)}\left(M_{\rho}\right)=(6.9 \pm 0.7) \cdot 10^{-3} & \left(\text { from }\left\langle r^{2}\right\rangle_{\mathrm{V}}^{\pi}\right) \\
L_{10}^{(0)}\left(M_{\rho}\right)=-(5.5 \pm 0.7) \cdot 10^{-3} & \left(\text { from } \pi^{-} \rightarrow e \bar{\nu}_{e} \gamma\right)
\end{array}
$$

We may employ these values for $L_{9}^{(0)}$ and $L_{10}^{(0)}$ in the two-loop quantities $G_{\pi}^{(2)}$ and $G_{K}^{(2)}$ of Eq. (9) because any error made is of still higher order. This is, however, not true for the $L_{10}^{(0)}$ dependence in $G^{(1)}$, for which consistency demands a two-loop evaluation. That is, suppose hypothetically that a physical amplitude existed which contained $L_{10}^{(0)}$, but no other counterterms to either one-loop or two-loop order. A phenomenological determination of $L_{10}^{(0)}$ carried out in a two-loop analysis would yield a value somewhat different from that obtained in a one-loop analysis. We would expect this shift in $L_{10}^{(0)}$ to be of the same order as the $p^{6}$ counterterms which comprise $G_{\mathrm{CT}}^{(2)}$ in Eq. (10), and so we are not justified a priori in ignoring it.

Of the $p^{6}$ counterterms, we have previously used spectral function sum rules to obtain the estimates,

\footnotetext{
${ }^{1}$ The reader is referred to Ref. [10] for a discussion of renormalization scheme dependence of the counterterms.
} 


$$
\begin{array}{ll}
Q_{\mathrm{V}}^{\overline{\mathrm{MS}}}\left(M_{\rho}\right)=(3.7 \pm 2.0) \cdot 10^{-5} & \text { (Ref. [15]) } \\
Q_{\mathrm{A}}^{\overline{\mathrm{MS}}}\left(M_{\rho}\right)=(1.4 \pm 0.5) \cdot 10^{-4} \quad \text { (Ref. [10) }
\end{array}
$$

where the result of Ref. [10] has been scaled down to $\mu=M_{\rho}$ in the above. The relatively large uncertainties in each of these reflects the paucity of existing data in the hypercharge channel. It is also possible to obtain estimates of certain counterterms via the assumption of resonance exchange saturation [17]. Thus, a reanalysis [18] of the paper by Jetter [19] on $\gamma \gamma \rightarrow \pi \pi$ and $\eta \rightarrow \pi \gamma \gamma$ yields

$$
\left.Q_{\mathrm{V}}^{\overline{\mathrm{MS}}}\right|_{\mathrm{res}}=F_{\pi}^{2} \frac{C_{S}^{\gamma} C_{S}^{m}}{M_{S}^{2}} \simeq \pm 3.0 \cdot 10^{-5},\left.\quad R_{\mathrm{V}}^{\overline{\mathrm{MS}}}\right|_{\mathrm{res}}=0
$$

Since the counterterm $Q_{\mathrm{V}}^{\overline{\mathrm{MS}}}$ receives contributions in the resonance exchange approach only from scalar exchange, its magnitude (the sign is not fixed) is rendered small. The resonance couplings $C_{S}^{\gamma}$ and $C_{S}^{m}$ are defined in Appendix D.2 of Ref. [20], and the result is seen to agree nicely with our sum rule determination cited in Eq. (17). As for $R_{\mathrm{V}}^{\overline{\mathrm{MS}}}$, although the contributions from low-lying resonances vanish, there will of course be continuum contributions. Even so, we disagree with the numerical estimates given in Ref. [19], and the vanishing result for $\left.R_{\mathrm{V}}^{\overline{\mathrm{MS}}}\right|_{\text {res }}$ implies that the interpretation of a large $\eta \rightarrow \pi \gamma \gamma$ amplitude given in Ref. 19 is not tenable.

\section{Analysis and Conclusions}

We are now ready to study the numerical implications of the DMO sum rule. To begin, let us temporarily avoid any use of counterterm inputs in order to get a result free of theoretical errors. Then using the conservative estimate of Eq. (12) for the DMO spectral integral $I_{\mathrm{DMO}}$, we obtain from the DMO sum rule the numerical constraint

$$
\begin{aligned}
& L_{10}^{(0)}\left(M_{\rho}\right)-\frac{M_{\pi}^{2}}{F_{\pi}^{2}}\left[Q_{\mathrm{A}}^{\overline{\mathrm{MS}}}\left(M_{\rho}\right)-Q_{\mathrm{V}}^{\overline{\mathrm{MS}}}\left(M_{\rho}\right)\right]-\frac{M_{\pi}^{2}+2 M_{K}^{2}}{F_{\pi}^{2}}\left[R_{\mathrm{A}}^{\overline{\mathrm{MS}}}\left(M_{\rho}\right)-R_{\mathrm{V}}^{\overline{\mathrm{MS}}}\left(M_{\rho}\right)\right] \\
& =-\frac{1}{4}\left[I_{\mathrm{DMO}}-\bar{G}^{(1)}\left(M_{\rho}\right)-\bar{G}^{(2)}\left(M_{\rho}\right)\right]=-0.0038 \pm 0.0004,
\end{aligned}
$$

where $\bar{G}^{(1)} \equiv G^{(1)}+4 L_{10}^{(0)}$ and $\bar{G}^{(2)} \equiv G^{(2)}-G_{\mathrm{CT}}^{(2)}$. The RHS of Eq. (19) will change in a known manner as the scale $\mu$ changes but the error bar, due exclusively to the uncertainty in the evaluation of $I_{\mathrm{DMO}}$, will remain the same. The value of the RHS is seen to be rather smaller in magnitude than the one-loop value $L_{10}^{(0)}\left(M_{\rho}\right) \simeq-0.0055$. This is due in large part to sizeable chiral logarithms present in $G_{\pi}^{(2)}$ and $G_{K}^{(2)}$. That is, the numerical contribution of $I_{\mathrm{DMO}}$ to the RHS (equal to -0.0066) is reduced via cancellation with the one-loop term $\bar{G}^{(1)}$ to -0.0054 and then via cancellation with the two-loop term $\bar{G}^{(2)}$ to the final value of -0.0038 . The latter reduction is about a $30 \%$ effect.

Can the contribution of $L_{10}^{(0)}\left(M_{\rho}\right)$ be disentangled from the mass corrections on the LHS of Eq. (19)? The interest in this question is underlined by the large numerical effect, just mentioned, the chiral logarithms have on the LHS of the DMO sum rule. From Eq. (19), one can already conclude that if $L_{10}^{(0)}\left(M_{\rho}\right)$ stays close to the one-loop determination cited in Eq. (16), the mass corrections involving the $p^{6}$ counterterms have to be both substantial 
and negative at scale $\mu=M_{\rho}$. Conversely, if these mass corrections turn out to be small at $\mu=M_{\rho}$, any two-loop analysis would need to yield a determination for $L_{10}^{(0)}\left(M_{\rho}\right)$ reduced by about $30 \%$ relative to its one-loop value.

The information gathered in Eqs. (17), (18) is not by itself sufficient to conclusively answer this question. Nevertheless it is instructive to investigate in more detail the individual contributions to the LHS of the DMO sum rule. Using the estimates of Eq. (17), the contribution of the Q-type counterterms is numerically found to be

$$
-\frac{4 M_{\pi}^{2}}{F_{\pi}^{2}}\left[Q_{\mathrm{A}}^{\overline{\mathrm{MS}}}\left(M_{\rho}\right)-Q_{\mathrm{V}}^{\overline{\mathrm{MS}}}\left(M_{\rho}\right)\right]=(9.3 \pm 5.0) \cdot 10^{-4}
$$

where we have added errors on $Q_{\mathrm{A}}^{\overline{\mathrm{MS}}}$ and $Q_{\mathrm{V}}^{\overline{\mathrm{MS}}}$ in quadrature. The Q-terms thus contribute approximately $3.5 \%$ to the value obtained phenomenologically for the RHS of the DMO sum rule. For $R_{\mathrm{V}}^{\overline{\mathrm{MS}}}$ we can employ the resonance saturation estimate of Eq. (18). No corresponding estimate is available, however, for $R_{\mathrm{A}}^{\overline{\mathrm{MS}}}$. A priori, such contributions proceed through exchange of scalar resonances as well, but the couplings of those resonances to two axialvector currents are not known experimentally. However, since both R-type counterterms are Zweig-rule suppressed, we expect these to be small compared to the Q-type counterterms.

There is, however, a well-known drawback of such resonance saturation estimates of counterterms. The method does not specify the scale at which the counterterm has to be taken. The generally accepted procedure is to employ the resonance mass as the relevant scale and to use the variation of the counterterm in a range between, say, $\mu=0.5 \rightarrow 1 \mathrm{GeV}$ as an estimate of the uncertainty of the method. In the DMO sum rule, the counterterms turn out to vary strongly with scale. This can be seen either by using Eqs. (15) or be read off from Table 1 where we give the two-loop contributions $G_{\pi}^{(2)}, G_{K}^{(2)}$ and $G_{\mathrm{NUM}}^{(2)}$ for three different scales $\mu$.

Table 1: Scale dependence

\begin{tabular}{l|rrr}
\hline \hline$\mu(\mathrm{GeV})$ & \multicolumn{1}{c}{0.50} & 0.77 & \multicolumn{1}{c}{1.00} \\
\hline$G_{\pi}^{(2)}(\mu)$ & 0.0018 & 0.0023 & 0.0026 \\
$G_{K}^{(2)}(\mu)$ & 0.0001 & 0.0042 & 0.0065 \\
$G_{\mathrm{NUM}}^{(2)}$ & -0.0003 & -0.0003 & -0.0003 \\
\hline $\operatorname{Sum}(\mu)$ & 0.0016 & 0.0062 & 0.0088 \\
\hline
\end{tabular}

For the range of scales between $\mu=0.5 \mathrm{GeV}$ and $\mu=1 \mathrm{GeV}$ the variation is 0.007 , or $27 \%$ of the RHS of the DMO sum rule. This variation with scale is of course counterbalanced by the $p^{6}$ counterterms. However, as just explained, if an estimate for these counterterms is used where one has no control over the scale at which the counterterm is fixed, the strong variation with scale translates into a large uncertainty in the determination of the LHS of the DMO sum rule. Consequently, it will be possible to disentangle $L_{10}^{(0)}\left(M_{\rho}\right)$ from the mass corrections on the LHS of the DMO sum rule only if one can perform an independent determination of $R_{\mathrm{A}}^{\overline{\mathrm{MS}}}-R_{\mathrm{V}}^{\overline{\mathrm{MS}}}$ directly from physical observables.

To close the discussion, we present some remarks concerning existing and future work in this direction. A related process in which $L_{10}^{(0)}$ contributes at order $p^{4}$ is the radiative pion decay $\pi \rightarrow e \bar{\nu}_{e} \gamma$. In this process, however, it is the combination $L_{9}^{(0)}+L_{10}^{(0)}$ which occurs, and 
the structure dependent part of the amplitude is actually used to determine this combination of $p^{4}$ counterterms. [ At order $p^{6}$, additional counterterms will contribute, some of them not occuring in the two-point functions considered here. Bijnens and Talavera have calculated the radiative pion decay amplitude to two-loop order [21], although in chiral SU(2). Using the hypotheses of resonance saturation (with just vector- and axialvector resonances), they estimate the $p^{6}$ counterterm contributions to be small. The sum of the two-loop contributions is large, however, leading to an enhancement of about $15 \%$ for $2 l_{5}^{r}-l_{6}^{r}$ at the scale $M_{\rho}$. The advantage of an $\mathrm{SU}(2)$ calculation is that mass corrections are always suppressed by factors of $M_{\pi}^{2}$. The large uncertainties due to scale-dependence of resonance saturated counterterms found in our SU(3) calculation are thus largely avoided. The comparsion of our results to the DMO sum rule in its $\mathrm{SU}(2)$ version will thus be an interesting avenue for future work. On the other hand, working in chiral $\mathrm{SU}(3)$ offers the possibility of studying additional processes with external currents/Goldstone Bosons containing strangeness. For instance, there are the radiative $K_{l 2}$ decays [22], where more precise data is expected in the near future [23]. However, because at one-loop order $K \rightarrow \ell \bar{\nu}_{\ell} \gamma$ involves the combination $L_{9}^{(0)}+L_{10}^{(0)}$, learning more about just $L_{10}^{(0)}$ will require the study of other processes in which $L_{9}^{(0)}$ enters separately. The meson form factors of vector current matrix elements, $\left\langle P\left|V_{\mu}\right| Q\right\rangle$, where $P, Q=\pi, K, \eta$, offer one possibility to study mass corrections to $L_{9}^{(0)}$. Partial results on a combination of such form factors have been reported recently [24]. It remains to be seen whether the mass corrections to the individual form factors can be disentangled from the contributions of the low energy constant $L_{9}^{(0)}$.

To conclude, we have used our recent two-loop calculation of isospin and hypercharge vector and axialvector current propagators in chiral perturbation theory to re-analyze the DMO sum rule. The resulting relation, valid to second order in the light quark masses, is summarized in Eqs. (8)-(10). Since we work in $\mathrm{SU}(3) \times \mathrm{SU}(3)$ chiral symmetry, both pion and kaon mass dependence is present. A phenomenological determination of the DMO spectral integral yielded a numerical constraint on the combination of $p^{4}$ and $p^{6}$ counterterms, given in Eq. (19), and prospects for extending the analysis were considered. As regards the overall question of how quickly the chiral expansion is converging, we find individual contributions at two-loop level to occur at roughly the $30 \%$ level.

\section{ACKNOWLEDGMENTS}

The research described here was supported in part by the National Science Foundation and by Schweizerischer Nationalfonds. We acknowledge useful conversations with J. Bijnens and J. Donoghue. We thank M. Knecht for allowing us to use an unpublished result [18].

\footnotetext{
${ }^{2} \mathrm{In} \mathrm{SU}(2)$ notation, the relevant combination of counterterms is $2 l_{5}^{r}-l_{6}^{r}$. Its relation to the $\mathrm{SU}(3)$ couplings is known only to one-loop order. [7] A direct comparison with our analysis is therefore not yet possible.
} 


\section{REFERENCES}

[1] S. Weinberg, Phys. Rev. Lett. 18 (1967) 507.

[2] T. Das, G.S. Guralnik, V.S. Mathur, F.E. Low and J.E. Young, Phys. Rev. Lett. 18 (1967) 759.

[3] T. Das, V.S. Mathur and S. Okubo, Phys. Rev. Lett. 19 (1967) 859.

[4] E.G. Floratos, S. Narison and E. de Rafael, Nucl. Phys. B155 (1979) 115.

[5] S. Narison and E. de Rafael, Nucl. Phys. B169 (1980) 253.

[6] J. Gasser and H. Leutwyler, Ann. Phys. 158 (1984) 142.

[7] J. Gasser and H. Leutwyler, Nucl. Phys. B250 (1985) 465.

[8] J.F. Donoghue and B.R. Holstein, Phys. Rev. D46 (1992) 4076.

[9] E. Golowich and J. Kambor, Nucl. Phys. B447, (1995) 373.

[10] E. Golowich and J. Kambor, 'Two-loop Analysis of Axialvector Current Propagators in Chiral Perturbation Theory', Univ. of Massachusetts preprint UMHEP-445 and Univ. of Zürich preprint ZU-TH 19/97, hep-ph/9710214.

[11] E. Braaten, S. Narison and A. Pich, Nucl. Phys. B373 (1992) 581; M. Jamin and M. Münz, Z. Phys. C60 (1993) 569.

[12] 'Chiral Sum Rules to Second Order in Quark Mass', E. Golowich and J. Kambor, Phys. Rev. Lett. (to be published); hep-ph/9707341.

[13] J.F. Donoghue and E. Golowich, Phys. Rev. D49 (1994) 1513.

[14] A. Höcker, 'Vector and Axialvector Spectral Functions and QCD', hep-ex/9703004.

[15] E. Golowich and J. Kambor, Phys. Rev. D53 (1996) 2651.

[16] See G. Ecker, in Chiral Dynamics: Theory and Experiment, Eds. A.M. Bernstein and B.R. Holstein, Springer (Berlin, Heidelberg) 1995.

[17] G. Ecker, J. Gasser, A. Pich and E. de Rafael, Nucl. Phys. B321 (1989) 311.

[18] J. Kambor and M. Knecht, unpublished.

[19] M. Jetter, Nucl. Phys. B459 (1996) 283.

[20] S. Bellucci, J. Gasser and M.E. Sainio, Nucl. Phys. B423 (1994) 80; ibid B431 (1994) 413 (Erratum).

[21] J. Bijnens and P. Talavera, Nucl. Phys. B489 (1997) 387.

[22] J. Bijnens, G. Ecker and J. Gasser, Nucl. Phys. B396 (1993) 81.

[23] E.g. see J. Bijnens et al, 'Semileptonic Kaon Decays', hep-ph/9411311, published in The Second DAథNE Physics Handbook, L. Maiani, G. Pancheri and N. Paver (Eds.), (INFN, Frascati, 1995).

[24] P. Post and K. Schilcher, preprint hep-ph/9701422. 